\title{
A definable nonstandard model of the reals
}

\author{
Vladimir Kanovei* Saharon Shelah ${ }^{\dagger}$
}

August 2003

\begin{abstract}
We prove, in ZFC, the existence of a definable, countably saturated elementary extension of the reals.
\end{abstract}

\section{Introduction}

It seems that it has been taken for granted that there is no distinguished, definable nonstandard model of the reals. (This means a countably saturated elementary extension of the reals.) Of course if $\mathbf{V}=\mathbf{L}$ then there is such an extension (just take the first one in the sense of the canonical well-ordering of $\mathbf{L}$ ), but we mean the existence provably in ZFC. There were good reasons for this: without Choice we cannot prove the existence of any elementary extension of the reals containing an infinitely large integer. ${ }^{1}{ }^{2}$ Still there is one.

Theorem 1 ( ZFC). There exists a definable, countably saturated extension ${ }^{*} \mathbb{R}$ of the reals $\mathbb{R}$, elementary in the sense of the language containing a symbol for every finitary relation on $\mathbb{R}$.

The problem of the existence of a definable proper elementary extension of $\mathbb{R}$ was communicated to one of the authors (Kanovei) by V. A. Uspensky.

A somewhat different, but related problem of unique existence of a nonstandard real line ${ }^{*} \mathbb{R}$ has been widely discussed by specialists in nonstandard analysis. ${ }^{3}$ Keisler notes in [3, $\S 11]$ that, for any cardinal $\kappa$, either inaccesible or satisfying $2^{\kappa}=\kappa^{+}$, there exists unique, up to isomorphism, $\kappa$-saturated nonstandard real line ${ }^{*} \mathbb{R}$ of cardinality $\kappa$, which means that a reasonable level of uniqueness modulo isomorphism can be

*Partial support of RFFI grant 03-01-00757 and DFG grant acknowledged.

${ }^{\dagger}$ Supported by The Israel Science Foundation. Publication 825 .

${ }^{1}$ In fact, from any nonstandard integer we can define a non-principal ultrafilter on $\mathbb{N}$, even a Lebesgue non-measurable set of reals [4, yet it is consistent with ZF (even plus Dependent Choices) that there are no such ultrafilters as well as non-measurable subsets of $\mathbb{R}[\mathbf{5}]$.

${ }^{2}$ It is worth to be mentioned that definable nonstandard elementary extensions of $\mathbb{N}$ do exist in ZF. For instance, such a model can be obtained in the form of the ultrapower $F / U$, where $F$ is the set of all arithmetically definable functions $f: \mathbb{N} \rightarrow \mathbb{N}$ while $U$ is a non-principal ultrafilter in the algebra $A$ of all arithmetically definable sets $X \subseteq \mathbb{N}$.

3 "What is needed is an underlying set theory which proves the unique existence of the hyperreal number system [...]" (Keisler [3, p. 229]). 
achieved, say, under GCH. Theorem 1 provides a countably saturated nonstandard real line ${ }^{*} \mathbb{R}$, unique in absolute sense by virtue of a concrete definable construction in ZFC. A certain modification of this example also admits a reasonable model-theoretic characterization up to isomorphism (see Section 4).

The proof of Theorem 1 is a combination of several known arguments. First of all (and this is the key idea), arrange all non-principal ultrafilters over $\mathbb{N}$ in a linear order $A$, where each ultrafilter appears repetitiously as $D_{a}, a \in A$. Although $A$ is not a well-ordering, we can apply the iterated ultrapower construction in the sense of [1. 6.5] (which is "a finite support iteration" in the forcing nomenclature), to obtain an ultrafilter $D$ in the algebra of all sets $X \subseteq \mathbb{N}^{A}$ concentrated on a finite number of axes $\mathbb{N}$. To define a $D$-ultrapower of $\mathbb{R}$, the set $F$ of all functions $f: \mathbb{N}^{A} \rightarrow \mathbb{R}$, also concentrated on a finite number of axes $\mathbb{N}$, is considered. The ultrapower $F / D$ is OD, thar is, ordinal-definable, actually, definable by an explicit construction in ZFC, hence, we obtain an OD proper elementary extension of $\mathbb{R}$. Iterating the $D$-ultrapower construction $\omega_{1}$ times in a more ordinary manner, i. e., with direct limits at limit steps, we obtain a definable countably saturated extension.

To make the exposition self-contained and available for a reader with only fragmentary knowledge of ultrapowers, we reproduce several well-known arguments instead of giving references to manuals.

\section{The ultrafilter}

As usual, $\mathfrak{c}$ is the cardinality of the continuum.

Ultrafilters on $\mathbb{N}$ hardly admit any definable linear ordering, but maps $a: \mathfrak{c} \rightarrow$ $\mathscr{P}(\mathbb{N})$, whose ranges are ultrafilters, readily do. Let $A$ consist of all maps $a: \mathfrak{c} \rightarrow$ $\mathscr{P}(\mathbb{N})$ such that the set $D_{a}=\operatorname{ran} a=\{a(\xi): \xi<\mathfrak{c}\}$ is an ultrafilter on $\mathbb{N}$. The set $A$ is ordered lexicographically: $a<_{\text {lex }} b$ means that there exists $\xi<\mathfrak{c}$ such that $a\lceil\xi=b\lceil\xi$ and $a(\xi)<b(\xi)$ in the sense of the lexicographical linear order $<$ on $\mathscr{P}(\mathbb{N})$ (in the sense of the identification of any $u \subseteq \mathbb{N}$ with its characterictic function).

For any set $u, \mathbb{N}^{u}$ denotes the set of all maps $f: u \rightarrow \mathbb{N}$.

Suppose that $u \subseteq v \subseteq A$.

If $X \subseteq \mathbb{N}^{v}$ then put $X \downarrow u=\{x \uparrow u: x \in X\}$.

If $Y \subseteq \mathbb{N}^{u}$ then put $Y \uparrow v=\left\{x \in \mathbb{N}^{v}: x \uparrow u \in Y\right\}$.

We say that a set $X \subseteq \mathbb{N}^{A}$ is concentrated on $u \subseteq A$, if $X=(X \downarrow u) \uparrow A$; in other words, this means the following:

$$
\forall x, y \in \mathbb{N}^{A}(x\lceil u=y\lceil u \Longrightarrow(x \in X \Longleftrightarrow y \in X))
$$

We say that $X$ is a set of finite support, if it is concentrated on a finite set $u \subseteq A$. The collection $\mathscr{X}$ of all sets $X \subseteq \mathbb{N}^{A}$ of finite support is closed under unions, intersections, complements, and differences, i. e., it is an algebra of subsets of $\mathbb{N}^{A}$. Note that if $(*)$ holds for finite sets $u, v \subseteq A$ then it also holds for $u \cap v$. (If $x \uparrow(u \cap v)=y \uparrow(u \cap v)$ then consider $z \in \mathbb{N}^{A}$ such that $z\lceil u=x \uparrow u$ and $z\lceil v=y\lceil v$.) It follows that for any $X \in \mathscr{X}$ there is a least finite $u=\|X\| \subseteq A$ satisfying $(*)$.

In the remainder, if $U$ is any subset of $\mathscr{P}(I)$, where $I$ is a given set, then $U i \Phi(i)$ (generalized quantifier) means that the set $\{i \in I: \Phi(i)\}$ belongs to $U$. 
The following definition realizes the idea of a finite iteration of ultrafilters. Suppose that $u=a_{1}<\cdots<a_{n} \subseteq A$ is a finite set. We put

$$
\begin{aligned}
D_{u} & =\left\{X \subseteq \mathbb{N}^{u}: D_{a_{n}} k_{n} \ldots D_{a_{2}} k_{2} D_{a_{1}} k_{1}\left(\left\langle k_{1}, k_{2}, \ldots, k_{n}\right\rangle \in X\right)\right\} ; \\
D & =\left\{X \in \mathscr{X}: X \downarrow\|X\| \in D_{\|X\|}\right\} .
\end{aligned}
$$

The following is quite clear.

Proposition 2. (i) $D_{u}$ is an ultrafilter on $\mathbb{N}^{u}$;

(ii) if $u \subseteq v \subseteq A, v$ finite, $X \subseteq \mathbb{N}^{u}$, then $X \in D_{u}$ iff $X \uparrow v \in D_{v}$;

(iii) $D \subseteq \mathscr{X}$ is an ultrafilter in the algebra $\mathscr{X}$;

(iv) if $X \in \mathscr{X}, u \subseteq A$ finite, and $\|X\| \subseteq u$, then $X \in D \Longleftrightarrow X \downarrow u \in D_{u}$.

\section{The ultrapower}

To match the nature of the algebra $\mathscr{X}$ of sets $X \subseteq \mathbb{N}^{A}$ of finite support, we consider the family $F$ of all $f: \mathbb{N}^{A} \rightarrow \mathbb{R}$, concentrated on some finite set $u \subseteq A$, in the sense that

$$
\forall x, y \in \mathbb{N}^{A}(x\lceil u=y\lceil u \Longrightarrow f(x)=f(y)) .
$$

As above, for any $f \in F$ there exists a least finite $u=\|f\| \subseteq A$ satisfying ( $\dagger$ ).

Let $\mathscr{R}$ be the set of all finitary relations on $\mathbb{R}$. For any $n$-ary relation $E \in \mathscr{R}$ and any $f_{1}, \ldots, f_{n} \in F$, define

$$
E^{D}\left(f_{1}, \ldots, f_{n}\right) \Longleftrightarrow D x \in \mathbb{N}^{A} E\left(f_{1}(x), \ldots, f_{n}(x)\right) .
$$

The set $X=\left\{x \in \mathbb{N}^{A}: E\left(f_{1}(x), \ldots, f_{n}(x)\right)\right\}$ is obviously concentrated on $u=\left\|f_{1}\right\| \cup$ $\cdots \cup\left\|f_{n}\right\|$, hence, it belongs to $\mathscr{X}$, and $\|X\| \subseteq u=\left\|f_{1}\right\| \cup \cdots \cup\left\|f_{n}\right\|$.

In particular, $f={ }^{D} g$ means that $D x \in \mathbb{N}^{A}(f(x)=g(x))$. The following is clear:

Proposition 3. $={ }^{D}$ is an equivalence relation on $F$, and any relation on $F$ of the form $E^{D}$ is $=^{D}$-invariant.

Put $[f]_{D}=\left\{g \in F: f={ }^{D} g\right\}$, and ${ }^{*} \mathbb{R}=F / D=\left\{[f]_{D}: f \in F\right\}$. For any $n$-ary $(n \geq 1)$ relation $E \in \mathscr{R}$, let ${ }^{*} E$ be the relation on ${ }^{*} \mathbb{R}$ defined as follows:

$$
{ }^{*} E\left(\left[f_{1}\right]_{D}, \ldots,\left[f_{n}\right]_{D}\right) \quad \text { iff } \quad E^{D}\left(f_{1}, \ldots, f_{n}\right) \quad \text { iff } \quad D x \in \mathbb{N}^{A} E\left(f_{1}(x), \ldots, f_{n}(x)\right) .
$$

The independence on the choice of representatives in the classes $\left[f_{i}\right]_{D}$ follows from Proposition 3. Put ${ }^{*} \mathscr{R}=\left\{{ }^{*} E: E \in \mathscr{R}\right\}$. Finally, for any $r \in \mathbb{R}$ we put ${ }^{*} r=\left[c_{r}\right]_{D}$, where $c_{r} \in F$ satisfies $c_{r}(x)=r, \forall x$.

Let $\mathscr{L}$ be the first-order language containing a symbol $E$ for any relation $E \in \mathscr{R}$. Then $\langle\mathbb{R} ; \mathscr{R}\rangle$ and $\left\langle{ }^{*} \mathbb{R} ;{ }^{*} \mathscr{R}\right\rangle$ are $\mathscr{L}$-structures.

Theorem 4. The map $r \longmapsto{ }^{*} r$ is an elementary embedding (in the sense of the language $\mathscr{L})$ of the structure $\langle\mathbb{R} ; \mathscr{R}\rangle$ into $\left\langle{ }^{*} \mathbb{R} ;{ }^{*} \mathscr{R}\right\rangle$. 
Proof. This is a routine modification of the ordinary argument. By $\mathscr{L}[F]$ we denote the extension of $\mathscr{L}$ by functions $f \in F$ used as parameters. It does not have a direct semantics, but if $\varphi$ is a formula of $\mathscr{L}[F]$ and $x \in \mathbb{N}^{A}$ then $\varphi[x]$ will denote the formula obtained by the substitution of $f(x)$ for any $f \in F$ which occurs in $\varphi$. Thus, $\varphi[x]$ is an $\mathscr{L}$-formula with parameters in $\mathbb{R}$.

Lemma 5 (Loš). For any closed $\mathscr{L}[F]$-formula $\varphi\left(f_{1}, \ldots, f_{n}\right)$ (all parameters $f_{i} \in F$ indicated), we have:

$$
\left\langle^{*} \mathbb{R} ;{ }^{*} \mathscr{R}\right\rangle \models \varphi\left(\left[f_{1}\right]_{D}, \ldots,\left[f_{n}\right]_{D}\right) \Longleftrightarrow D x\left(\langle\mathbb{R} ; \mathscr{R}\rangle \models \varphi\left(f_{1}, \ldots, f_{n}\right)[x]\right) .
$$

Proof. We argue by induction on the logic complexity of $\varphi$. For $\varphi$ an atomic relation $E\left(f_{1}, \ldots, f_{n}\right)$, the result follows by the definition of ${ }^{*} E$. The only notable induction step is $\exists$ in the direction $\Longleftarrow$. Suppose that $\varphi$ is $\exists y \psi\left(y, f_{1}, \ldots, f_{n}\right)$, and

$$
D x\left(\langle\mathbb{R} ; \mathscr{R}\rangle \models \varphi\left(f_{1}, \ldots, f_{n}\right)[x]\right), \quad \text { that is, } \quad D x\left(\langle\mathbb{R} ; \mathscr{R}\rangle \models \exists y \psi\left(y, f_{1}, \ldots, f_{n}\right)[x]\right) .
$$

Obviously there exists a function $f \in F$, concentrated on $u=\left\|f_{1}\right\| \cup \cdots \cup\left\|f_{n}\right\|$, such that, for any $x \in \mathbb{N}^{A}$, if there exists a real $y$ satisfying $\langle\mathbb{R} ; \mathscr{R}\rangle \models \psi\left(y, f_{1}, \ldots, f_{n}\right)[x]$, then $y=f(x)$ also satisfies this formula, i. e., $\langle\mathbb{R} ; \mathscr{R}\rangle \models \psi\left(f, f_{1}, \ldots, f_{n}\right)[x]$. Formally,

$$
\forall x \in \mathbb{N}^{A}\left(\exists y \in \mathbb{R}\left(\langle\mathbb{R} ; \mathscr{R}\rangle \models \psi\left(y, f_{1}, \ldots, f_{n}\right)[x]\right) \Longrightarrow\langle\mathbb{R} ; \mathscr{R}\rangle \models \psi\left(f, f_{1}, \ldots, f_{n}\right)[x]\right) .
$$

This implies $D x\left(\langle\mathbb{R} ; \mathscr{R}\rangle \models \psi\left(f, f_{1}, \ldots, f_{n}\right)[x]\right)$. Then, by the inductive assumption, $\left\langle{ }^{*} \mathbb{R} ;{ }^{*} \mathscr{R}\right\rangle \models \psi\left([f]_{D},\left[f_{1}\right]_{D}, \ldots,\left[f_{n}\right]_{D}\right)$, hence $\left\langle{ }^{*} \mathbb{R} ;{ }^{*} \mathscr{R}\right\rangle \models \varphi\left(\left[f_{1}\right]_{D}, \ldots,\left[f_{n}\right]_{D}\right)$, as required.

$\square$ (Lemma)

To accomplish the proof of Theorem [4. consider a closed $\mathscr{L}$-formula $\varphi\left(r_{1}, \ldots, r_{n}\right)$ with parameters $r_{1}, \ldots, r_{n} \in \mathbb{R}$. We have to prove the equivalence

$$
\langle\mathbb{R} ; \mathscr{R}\rangle \models \varphi\left(r_{1}, \ldots, r_{n}\right) \Longleftrightarrow\left\langle{ }^{*} \mathbb{R} ;{ }^{*} \mathscr{R}\right\rangle \models \varphi\left({ }^{*} r_{1}, \ldots,{ }^{*} r_{n}\right) .
$$

Let $f_{i}=c_{r_{i}}$, thus, $f_{i} \in F$ and $f_{i}(x)=r_{i}, \forall x$. Obviously $\varphi\left(f_{1}, \ldots, f_{n}\right)[x]$ coincides with $\varphi\left(r_{1}, \ldots, r_{n}\right)$ for any $x \in \mathbb{N}^{A}$, hence $\varphi\left(r_{1}, \ldots, r_{n}\right)$ is equivalent to $D x \varphi\left(f_{1}, \ldots, f_{n}\right)[x]$. On the other hand, by definition, ${ }^{*} r_{i}=\left[f_{i}\right]_{D}$. Now the result follows by Lemma 5 ,

\section{The iteration}

Theorem 4 yields a definable proper elementary extension $\left\langle{ }^{*} \mathbb{R} ;{ }^{*} \mathscr{R}\right\rangle$ of the structure $\langle\mathbb{R} ; \mathscr{R}\rangle$. Yet this extension is not countably saturated due to the fact that the ultrapower ${ }^{*} \mathbb{R}$ was defined with maps concentrated on finite sets $u \subseteq A$ only. To fix this problem, we iterate the extension used above $\omega_{1}$-many times.

Suppose that $\langle M ; \mathscr{M}\rangle$ is an $\mathscr{L}$-structure, so that $\mathscr{M}$ consists of finitary relations on a set $M$, and for any $E \in \mathscr{R}$ there is a relation $E^{\mathscr{M}} \in \mathscr{M}$ of the same arity, associated with $E$. Let $F_{M}$ be the set of all maps $f: \mathbb{N}^{A} \rightarrow M$ concentrated on finite sets $u \subseteq A$. The structure $F_{M} / D=\left\langle{ }^{*} M ; \mathscr{M}\right\rangle$, defined as in Section 2, but with the modified $F$, will be called the $D$-ultrapower of $\langle M ; \mathscr{M}\rangle$. Theorem 4 remains true in this general setting: the map $x \longmapsto{ }^{*} x(x \in M)$ is an elementary embedding of $\langle M ; \mathscr{M}\rangle$ in $\left\langle{ }^{*} M ; \mathscr{M}\right\rangle$.

We define a sequence of $\mathscr{L}$-structures $\left\langle M_{\alpha} ; \mathscr{M}_{\alpha}\right\rangle, \alpha \leq \omega_{1}$, together with a system of elementary embeddings $e_{\alpha \beta}:\left\langle M_{\alpha} ; \mathscr{M}_{\alpha}\right\rangle \rightarrow\left\langle M_{\beta} ; \mathscr{M}_{\beta}\right\rangle, \alpha<\beta \leq \omega_{1}$, so that 
(i) $\left\langle M_{0} ; \mathscr{M}_{0}\right\rangle=\langle\mathbb{R} ; \mathscr{R}\rangle$;

(ii) $\left\langle M_{\alpha+1} ; \mathscr{M}_{\alpha+1}\right\rangle$ is the $D$-ultrapower of $\left\langle M_{\alpha} ; \mathscr{M}_{\alpha}\right\rangle$, that is, $\left\langle M_{\alpha+1} ; \mathscr{M}_{\alpha+1}\right\rangle=$ $F_{\alpha} / D$, where $F_{\alpha}=F_{M_{\alpha}}$ consists of all functions $f: \mathbb{N}^{A} \rightarrow M_{\alpha}$ concentrated on finite sets $u \subseteq A$. In addition, $e_{\alpha, \alpha+1}$ is the associated ${ }^{*}$-embedding $\left\langle M_{\alpha} ; \mathscr{M}_{\alpha}\right\rangle \rightarrow$ $\left\langle M_{\alpha+1} ; \mathscr{M}_{\alpha+1}\right\rangle$, while $e_{\gamma, \alpha+1}=e_{\alpha, \alpha+1} \circ e_{\gamma \alpha}$ for any $\gamma<\alpha$ (in other words, $e_{\gamma, \alpha+1}(x)=e_{\alpha, \alpha+1}\left(e_{\gamma \alpha}(x)\right)$ for all $\left.x \in M_{\alpha}\right)$;

(iii) if $\lambda \leq \omega_{1}$ is a limit ordinal then $\left\langle M_{\lambda} ; \mathscr{M}_{\lambda}\right\rangle$ is the direct limit of the structures $\left\langle M_{\alpha} ; \mathscr{M}_{\alpha}\right\rangle, \alpha<\lambda$. This can be achieved by the following steps:

(a) $M_{\lambda}$ is defined as the set of all pairs $\langle\alpha, x\rangle$ such that $x \in M_{\alpha}$ and $x \notin \operatorname{ran} e_{\gamma \alpha}$ for all $\gamma<\alpha$.

(b) If $E \in \mathscr{R}$ is an $n$-ary relation symbol then we define an $n$-ary relation $E_{\lambda}$ on $M_{\lambda}$ as follows. Suppose that $\mathbf{x}_{i}=\left\langle\alpha_{i}, x_{i}\right\rangle \in M_{\lambda}$ for $i=1, \ldots, n$. Let $\alpha=\sup \left\{\alpha_{1}, \ldots, \alpha_{n}\right\}$ and $z_{i}=e_{\alpha_{i}, \alpha}\left(x_{i}\right)$ for every $i$, so that $\alpha_{i} \leq \alpha<\lambda$ and $z_{i} \in M_{\alpha}$. (Note that if $\alpha_{i}=\alpha$ then $e_{\alpha_{i}, \alpha}$ is the identity.) Define $E_{\lambda}\left(\mathbf{x}_{1}, \ldots, \mathbf{x}_{n}\right)$ iff $\left\langle M_{\alpha} ; \mathscr{M}_{\alpha}\right\rangle \models E\left(z_{1}, \ldots, z_{n}\right)$.

(c) Put $\mathscr{M}_{\lambda}=\left\{E_{\lambda}: E \in \mathscr{R}\right\}-$ then $\left\langle M_{\lambda} ; \mathscr{M}_{\lambda}\right\rangle$ is an $\mathscr{L}$-structure.

(d) Define an embedding $e_{\alpha \lambda}: M_{\alpha} \rightarrow M_{\lambda}(\alpha<\lambda)$ as follows. Consider any $x \in M_{\alpha}$. If there is a least $\gamma<\alpha$ such that there exists an element $y \in M_{\gamma}$ with $x=e_{\gamma \alpha}(y)$ then let $e_{\alpha \lambda}(x)=\langle\gamma, y\rangle$. Otherwise put $e_{\alpha \lambda}(x)=\langle\alpha, x\rangle$.

A routine verification of the following is left to the reader.

Proposition 6. If $\alpha<\beta \leq \omega_{1}$ then $e_{\alpha \beta}$ is an elementary embedding of $\left\langle M_{\alpha} ; \mathscr{M}_{\alpha}\right\rangle$ to $\left\langle M_{\beta} ; \mathscr{M}_{\beta}\right\rangle$.

Note that the construction of the sequence of models $\left\langle M_{\alpha} ; \mathscr{M}_{\alpha}\right\rangle$ is definable, hence, so is the last member $\left\langle M_{\omega_{1}} ; \mathscr{M}_{\omega_{1}}\right\rangle$ of the sequence. It remains to prove that the $\mathscr{L}$ structure $\left\langle M_{\omega_{1}} ; \mathscr{M}_{\omega_{1}}\right\rangle$ is countably saturated.

This is also a simple argument. Suppose that, for any $k, \varphi_{k}\left(p_{k}, x\right)$ is an $\mathscr{L}$-formula with a single parameter $p_{k} \in M_{\omega_{1}}$ (the case of many parameters does not essentially differ from the case of one parameter), and there exists an element $x_{k} \in M_{\omega_{1}}$ such that $\bigwedge_{i \leq k} \varphi_{i}\left(p_{i}, x_{k}\right)$ is true in $\left\langle M_{\omega_{1}} ; \mathscr{M}_{\omega_{1}}\right\rangle$ - in other words, we have $\left\langle M_{\omega_{1}} ; \mathscr{M}_{\omega_{1}}\right\rangle \models$ $\varphi_{i}\left(p_{i}, x_{k}\right)$ whenever $k \geq i$. Fix an ordinal $\gamma<\omega_{1}$ such that for any $k, i$ there exist (then obviously unique) $y_{k}, q_{i} \in M_{\gamma}$ with $x_{k}=e_{\gamma \omega_{1}}\left(y_{k}\right)$ and $p_{i}=e_{\gamma \omega_{1}}\left(q_{i}\right)$. Then $\varphi_{i}\left(q_{i}, y_{k}\right)$ is true in $\left\langle M_{\gamma} ; \mathscr{M}_{\gamma}\right\rangle$ whenever $k \geq i$.

Fix $a \in A$ such that $D_{a}$ is a non-principal ultrafilter, that is, all cofinite subsets of $\mathbb{N}$ belong to $D_{a}$. Consider the structure $\left\langle M_{\gamma+1} ; \mathscr{M}_{\gamma+1}\right\rangle$ as the $D$-ultrapower of $\left\langle M_{\gamma} ; \mathscr{M}_{\gamma}\right\rangle$. The corresponding set $F_{\gamma}$ consists of all functions $f: \mathbb{N}^{A} \rightarrow M_{\gamma}$ concentrated on finite sets $u \subseteq A$. In particular, the map $f(x)=y_{k}$ whenewer $x(a)=k$ belongs to $F_{\gamma}$. As any set of the form $\{k: k \geq i\}$ belongs to $D_{a}$, we have $D_{a} k\left(\left\langle M_{\gamma} ; \mathscr{M}_{\gamma}\right\rangle \models \varphi_{i}\left(q_{i}, y_{k}\right)\right)$, that is, $D x \in \mathbb{N}^{A}\left(\left\langle M_{\gamma} ; \mathscr{M}_{\gamma}\right\rangle \models \varphi_{i}\left(q_{i}, f\right)[x]\right)$, for any $i \in \mathbb{N}$. It follows, by Lemma 5 , that $\varphi_{i}\left({ }^{*} q_{i}, \mathbf{y}\right)$ holds in $\left\langle M_{\gamma+1} ; \mathscr{M}_{\gamma+1}\right\rangle$ for any $i$, where ${ }^{*} q_{i}=e_{\gamma, \gamma+1}\left(q_{i}\right) \in M_{\gamma+1}$ while $\mathbf{y}=[f]_{D} \in M_{\gamma+1}$ is the $D$-equivalence class of $f$ in $F_{\gamma}$. Put $\mathbf{x}=e_{\gamma+1, \omega_{1}}(\mathbf{y})$; then $\varphi_{i}\left(p_{i}, \mathbf{x}\right)$ is true in $\left\langle M_{\omega_{1}} ; \mathscr{M}_{\omega_{1}}\right\rangle$ for any $i$ because obviously $p_{i}=e_{\gamma+1, \omega_{1}}\left({ }^{*} q_{i}\right), \forall i$.

(Theorem [1) 


\section{$4 \quad$ Varia}

By appropriate modifications of the constructions, the following can be achieved:

1. For any given infinite cardinal $\kappa$, a $\kappa$-saturated elementary extension of $\mathbb{R}$, definable with $\kappa$ as the only parameter of definition.

2. A special elementary extension of $\mathbb{R}$, of as large cardinality as desired. For instance, take, in stage $\alpha$ of the construction considered in Section 3, ultrafilters on $\beth_{\alpha}$. Then the result will be a definable special structure of cardinality $\beth_{\omega_{1}}$. Recall that special models of equal cardinality are isomorphic [1, Theorem 5.1.17]. Therefore, such a modification admits an explicit model-theoretical characterization up to isomorphism.

3. A class-size definable elementary extension of $\mathbb{R}, \kappa$-saturated for any cardinal $\kappa$.

4. A class-size definable elementary extension of the whole set universe, $\kappa$-saturated for any cardinal $\kappa$. (Note that this cannot be strengthened to Ord-saturation, i. e., saturation with respect to all class-size families. For instance, Ord ${ }^{M}$-saturated elementary extensions of a minimal transitive model $M \models \mathbf{Z F C}$, definable in $M$, do not exist — see [2, Theorem 2.8].)

The authors thank the anonimous referee for valuable comments and corrections.

\section{References}

[1] C. C. Chang and H. J. Keisler, Model Theory, 3rd ed., North Holland, Amsterdam, 1992, xiv +650 pp. (Studies in logic and foundations of mathematics, 73).

[2] V. Kanovei and M. Reeken, Internal approach to external sets and universes, part 1, Studia Logica, 1995, 55, no. 2, pp. 229-257.

[3] H. J. Keisler, The hyperreal line, in P. Erlich (ed.) Real numbers, generalizations of reals, and theories of continua, Kluwer Academic Publishers, 1994, pp. 207-237.

[4] W. A. J. Luxemburg, What is nonstandard analysis? Amer. Math. Monthly 1973, 80 (Supplement), pp. 38-67.

[5] R. M. Solovay, A model of set theory in which every set of reals is Lebesgue measurable, Ann. of Math. 1970, 92, pp. 1-56.

Vladimir Kanovei

Institute for information transmission problems (IPPI), Russian academy of sciences, Bol. Karetnyj Per. 19, Moscow 127994, Russia

E-mail address : kanovei@mccme.ru

Saharon Shelah

Institute of Mathematics, The Hebrew University of Jerusalem, 91904 Jerusalem, Israel, and Department of Mathematics, Rutgers University, New Brunswick, NJ 08854, USA E-mail address : shelah@math.huji.ac.il

URL: http://www.math.rutgers.edu/ ${ }^{\sim}$ shelah 